\title{
Similarities and Differences among Protein Dynamics Studied by Variable Temperature Nuclear Magnetic Resonance Relaxation
}

\author{
Baptiste Busi, Jayasubba Reddy Yarava, Andrea Bertarello, François Freymond, Wiktor Adamski, \\ Damien Maurin, Matthias Hiller, Hartmut Oschkinat, Martin Blackledge, and Lyndon Emsley*
}

Cite This: J. Phys. Chem. B 2021, 125, 2212-2221

Read Online

\section{ACCESS \\ Џlll Metrics \& More \\ Article Recommendations \\ Supporting Information}

ABSTRACT: Understanding and describing the dynamics of proteins is one of the major challenges in biology. Here, we use multifield variable-temperature NMR longitudinal relaxation $\left(R_{1}\right)$ measurements to determine the hierarchical activation energies of motions of four different proteins: two small globular proteins (GB1 and the SH3 domain of $\alpha$-spectrin), an intrinsically disordered protein (the C-terminus of the nucleoprotein of the Sendai virus, Sendai Ntail), and an outer membrane protein (OmpG). The activation energies map the motions occurring in the side chains, in the backbone, and in the hydration shells of the proteins. We were able to identify similarities and differences in the average motions of the proteins. We find that the NMR relaxation properties of the four

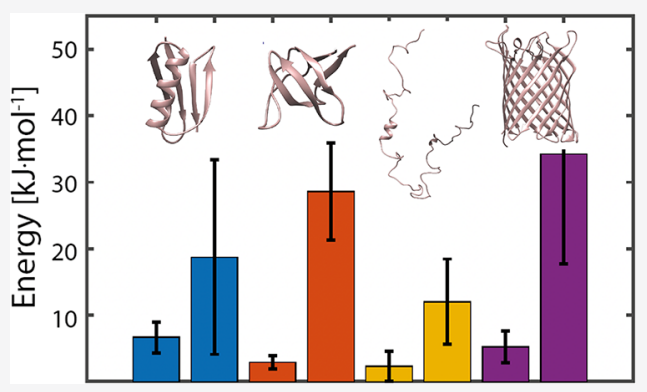
proteins do share similar features. The data characterizing average backbone motions are found to be very similar, the same for methyl group rotations, and similar activation energies are measured. The main observed difference occurs for the intrinsically disordered Sendai Ntail, where we observe much lower energy of activation for motions of protons associated with the protein-solvent interface as compared to the others. We also observe variability between the proteins regarding side chain ${ }^{15} \mathrm{~N}$ relaxation of lysine residues, with a higher activation energy observed in OmpG. This hints at strong interactions with negatively charged lipids in the bilayer and provides a possible mechanistic clue for the "positive-inside" rule for helical membrane proteins. Overall, these observations refine the understanding of the similarities and differences between hierarchical dynamics in proteins.

\section{INTRODUCTION}

Proteins are involved in virtually all biological processes, and the understanding of their atomic-level dynamics is essential to develop structure-function relationships. ${ }^{1-3}$ In particular, proteins show a series of different thermally activated motions, and function is a result of their interplay. Understanding the hierarchy of these motions is thus of high importance in linking dynamics to function.

The timescale of protein dynamics spans over several orders of magnitude, and the number of potential degrees of freedom is high, which makes the characterization of the individual modes a key challenge. ${ }^{4-7}$ For this purpose, a range of techniques were applied, such as differential scanning calorimetry (DSC), ${ }^{8,9}$ terahertz spectroscopy, ${ }^{10,11}$ Mössbauer spectroscopy, ${ }^{12,13}$ dielectric spectroscopy, ${ }^{8,12}$ Förster resonance energy transfer (FRET), ${ }^{14}$ X-ray crystallography, ${ }^{1,15,16}$ neutron scattering, ${ }^{17-19}$ and nuclear magnetic resonance (NMR). ${ }^{20-23}$ Variable-temperature studies have detected apparent transitions that have been related to the successive onset of dominant dynamic modes at temperatures ranging from 180 to $250 \mathrm{~K}^{8,11,24-28}$

NMR relaxation times are the tool of choice to determine dynamics at the atomic level, and they can yield accurate descriptions of timescales and amplitudes of motions in both

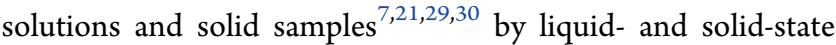
NMR, respectively. ${ }^{31,32}$ However, previous studies were mostly set out at room temperature. Investigation of dynamics in the cryogenic temperature range is only feasible by solid-state NMR. Recently, Lewandowski et al. ${ }^{20}$ presented a solid-state NMR approach to study the hierarchy of protein motions by measuring a set of 13 different ${ }^{1} \mathrm{H},{ }^{15} \mathrm{~N}$, and ${ }^{13} \mathrm{C} \mathrm{NMR}$ relaxation rates in the protein GB1 over a temperature range from 105 to $280 \mathrm{~K}$. The rates are affected by motions occurring on fast (ps) and slow (ns) timescales and in different portions of the system, ${ }^{20}$ and the data were analyzed by fitting the rates to a sum of spectral density functions related to Arrheniusdependent terms, which describe the superposition of distinct motional modes at different locations, and with different timescales and activation energies. The approach was subsequently extended to multiple-field measurements and allows a more precise description of the timescale and energies

Received: November 11, 2020

Revised: February 16, 2021

Published: February 26, 2021 
driving the backbone, side chain, and solvent motions in GB1. ${ }^{33}$ These studies described the apparent so-called "dynamical transitions" in terms of distinct activation energies of different dynamic modes. As an example, backbone amide protons experience both librational and collective anisotropic motions with low and high activation energies. The observed dynamical transition corresponds to the temperature at which the higher activation energy motion begins to dominate.

Despite large differences in both the structure and function among proteins of different families and despite the inevitable presence of dynamic heterogeneity at the local level due to the presence of specific binding sites or loops, for example, some features of protein dynamics are postulated to be "universal" properties. These include, for example, the coupling between protein regions and the solvent, which causes a second-order dynamic transition. ${ }^{12}$

In order to strive toward the determination of general principles of protein motion, we study overall protein dynamics in the absence of Brownian motion by measuring relaxation parameters averaged over different sites and thus evaluated at the full protein level.

Here, we present a comparative study of the similarities and differences in activation energies of four different proteins in three principally different kinds of sample conditions, using the variable-temperature variable-field relaxation approach. Specifically, we study microcrystalline preparations of two small globular proteins, GB1 and the SH3 domain of $\alpha$-spectrin, which appear as a solid at all temperatures. The soluble Sendai Ntail, which is intrinsically disordered, is dissolved in buffer and behaves as a solid upon sample freezing. The membrane protein OmpG, which is incorporated into lipid bilayers composed of Escherichia coli lipids and thus is also accessible to solid-state NMR methods at all temperatures. We measure ${ }^{1} \mathrm{H}$ $\mathrm{R}_{1}$ from ${ }^{1} \mathrm{H}$ spectra, ${ }^{1} \mathrm{H} \mathrm{R}_{1}$ on ${ }^{13} \mathrm{C}$ through cross polarization (CP), methyl ${ }^{13} \mathrm{C}_{1}$, amide ${ }^{15} \mathrm{~N} \mathrm{R}_{1}$, and lysine ${ }^{15} \mathrm{~N}_{\zeta} \mathrm{R}_{1}$ at multiple magnetic fields (from 400 to $900 \mathrm{MHz}$ ) and at temperatures in the range of 100 to $300 \mathrm{~K}$. The rates are analyzed to extract activation energies for the different modes, and the similarities and differences between the observed motions are discussed.

\section{METHODS}

Sample Preparation. GB1. The uniformly ${ }^{13} \mathrm{C},{ }^{15} \mathrm{~N}$ labelled, microcrystalline GB1 sample was purchased from Cortecnet. Their procedure was performed according to the following: ${ }^{34}$ First, the buffer was replaced by extensive dialysis against $50 \mathrm{mM}$ sodium phosphate buffer ( $\mathrm{pH}$ 5.5). The protein was concentrated to $25 \mathrm{mg} \cdot \mathrm{mL}^{-1}$. A mixture of $1: 2$ isopropanol:methyl-2-4-pentane-diol was used for precipitation of the protein by adding 3 volumes of the mixture for 1 volume of protein solution, which gives the sample the appearance of a milky solution. ${ }^{34}$ The obtained microcrystals are then introduced in the $3.2 \mathrm{~mm}$ rotor by ultracentrifugation using a Giotto Biotech device $\left(100,000 \mathrm{~g}, 4{ }^{\circ} \mathrm{C}\right)$ in order to sediment most of the microcrystals and remove as much as possible of the supernatant. ${ }^{35}$ The rotor was stored at $4{ }^{\circ} \mathrm{C}$ between experiment sessions.

SH3. The SH3 protein was overexpressed and purified as described by Castellani et al. ${ }^{36}$ Crystals were grown in a large scale sitting drop $(500 \mu \mathrm{L})$ with the hanging drop vapor diffusion method using $100 \mathrm{mM}$ ammonium sulphate titrated to a $\mathrm{pH}$ value or shift from 3.5 to 7.5 . The sample is then centrifugated three times on a bench Eppendorf centrifuge to remove all the excess water.

Ice crystal formation could potentially be an issue here, since there is no specific cryoprotectant, and the channels within the SH3 crystal structure are fairly large, but we did not see any changes in the sample after one full temperature cycle, as shown in Figure 1.

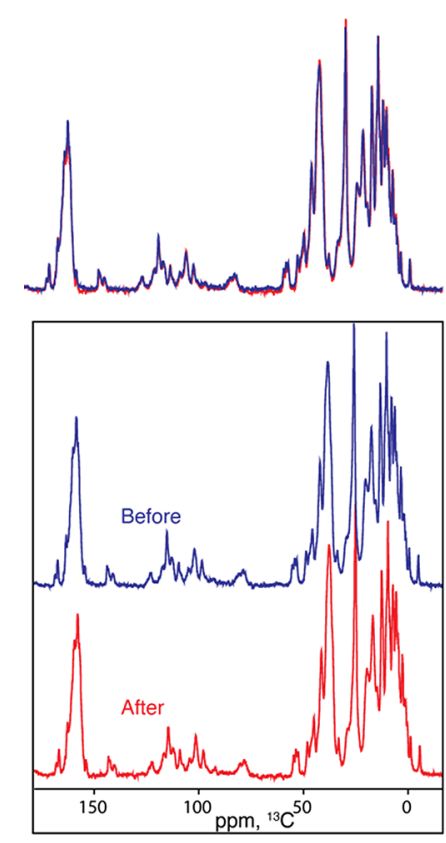

Figure 1. Comparison of $1 \mathrm{D}{ }^{13} \mathrm{C}$ CPMAS spectra of $\mathrm{SH} 3$ before and after one temperature cycle $(285 \mathrm{~K} \rightarrow 100 \mathrm{~K} \rightarrow 285 \mathrm{~K})$.

Sendai Ntail. The Sendai Ntail fragment was expressed as described previously by Blanchard et al. ${ }^{37}$ The solution contained approximately $750 \mu \mathrm{M}$ of protein with, at $\mathrm{pH} 6$, $50 \mathrm{mM}$ sodium phosphate and $500 \mathrm{mM} \mathrm{NaCl}$. The solution was flash frozen inside the rotor in liquid $\mathrm{N}_{2}$ before measurements.

OmpG. Expression was as described by Hiller et al. ${ }^{38}$ For refolding, purified OmpG was diluted into a buffer containing $n$-dodecyl- $\beta$-D-maltoside (DDM) $(1 \mathrm{mM})$ and L-arginine $(0.6$ $\mathrm{M})$ at $\mathrm{pH}$ 8.8. The refolded protein was washed and concentrated with buffer containing $10 \mathrm{~mm}$ Tris $-\mathrm{HCl}, \mathrm{pH}$ 8.0, and DDM (1 mM) to a final concentration of $1-2 \mathrm{mg}$. $\mathrm{mL}^{-1}$. The detergent concentration was reduced by binding OmpG to a Resource-Q column and washing with 3 column volumes of buffer containing $10 \mathrm{mM}$ Tris $-\mathrm{HCl}, \mathrm{pH} 8.0$, and DDM (0.4 mM). OmpG was eluted with $\mathrm{NaCl}(0.3 \mathrm{M})$ and concentrated by a centrifugal filter device to a final concentration of $3 \mathrm{mg} \cdot \mathrm{mL}^{-1}$.

Refolded OmpG was reconstituted into lipid bilayers. For this purpose, an E. coli total lipid chloroform extract $(20 \mathrm{mg}$ ) was dried in a nitrogen stream. The resulting lipid film was dissolved in buffer A comprising $10 \mathrm{mM}$ Tris- $\mathrm{HCl}, \mathrm{pH}$ 8.0, and $n$-octyl- $\beta$-D-glucopyranoside $(34 \mathrm{mM})$. Aliquots of this lipid solution and refolded OmpG $\left(2 \mathrm{mg} \cdot \mathrm{mL}^{-1}\right)$ were mixed to yield a lipid-to-protein ratio (LPR) of 1:2 (w/w). For 2D crystallization, the detergent was removed by dialysis (dialysis tube cutoff of $25 \mathrm{kDa}$; Roth, Karlsruhe, Germany) at $20{ }^{\circ} \mathrm{C}$ against buffer $\mathrm{B}(5 \mathrm{~L}, 20 \mathrm{mM}$ Tris $-\mathrm{HCl}, \mathrm{pH} 7.0,25 \mathrm{~mm}$ 
$\mathrm{MgCl}_{2}, 2.3 \mathrm{mM} \mathrm{NaN} 3$, and $150 \mathrm{mM} \mathrm{NaCl}$ ) for 6 to 7 weeks. The dialysis buffer was changed every 5 days.

No specific cryoprotectant was used. However, the sample was only subjected to one series of measurements with a decreasing temperature. Two samples were used, one for the experiments at 279.0,278.3,269.7, and $260.5 \mathrm{~K}$, and the other is for the rest.

NMR Experiments. All experiments were performed on an LT-MAS $3.2 \mathrm{~mm}$ probe in a triple resonance mode $\left({ }^{1} \mathrm{H},{ }^{15} \mathrm{~N}\right.$, ${ }^{13} \mathrm{C}$ ) at $10 \mathrm{kHz}$ spinning speed. Further details are included in the Supporting Information.

\section{RESULTS}

Samples. The four proteins are known from literature and were prepared according to published procedures. Streptococcus sp. GB1 (56 amino acids or aa), an antibody-binding protein, was previously used to introduce the methodology used here and to assess the reproducibility of the experiments and the robustness of the model. ${ }^{33}$ The protein was studied as a microcrystalline precipitate in a 3:1 $\mathrm{H}_{2} \mathrm{O}$ :methyl-2-4-pentanediol (MPD) molar ratio, with traces of isopropanol (IPA). All GB1 data shown here are reproduced from ref. ${ }^{33}$

The SH3 domain of chicken $\alpha$-spectrin (62 amino acids) is studied as microcrystals grown from $\mathrm{H}_{2} \mathrm{O}$, without a cryoprotectant, and served as a test case for a MAS structure determination methodology. ${ }^{36}$ Both samples have the characteristics that the crystal lattice is expected to influence local dynamics (e.g., compared to solution) and reduce solvent accessibility in addition to restriction in the hydrophobic core, particularly in crystal contact areas.

The Ntail of the nucleoprotein of the Sendai virus, referred to as Sendai Ntail, is an intrinsically disordered protein (IDP) with 126 amino acids. It was studied as a frozen, lowconcentration $(750 \mu \mathrm{M})$ solution. The protein has no hydrophobic core, and the whole protein may be considered as solvent exposed.

E. coli OmpG is a membrane protein, with 280 amino acids after removal of the signal peptide, here inserted into a lipid bilayer prepared from the E.coli total lipid extract with a lipidto-protein ratio of $1: 2(\mathrm{w} / \mathrm{w})$.

All proteins were uniformly ${ }^{13} \mathrm{C},{ }^{15} \mathrm{~N}$ labelled, except one batch of Sendai Ntail samples that was only ${ }^{13} \mathrm{C}$-labelled. The water and lipid contents of the samples packed into the rotors were evaluated qualitatively through signal integrals. The water-to-protein-to-MPD/IPA ratio in the GB1 sample was 1:0.03:0.59. For the $\mathrm{SH} 3$ sample, the water:protein ratio was 1:0.04. The water:protein ratio in the Sendai Ntail sample was not measurable and was above 1:0.01. The original sample in this case is unchanged by sample packing.

Two samples of OmpG from E. coli were used for the measurements. The water:lipid:protein ratio in the first sample was 1:0.06:0.03 and in the second sample was 1:1.17:0.02. However, despite the differences in composition (probably due to rotor packing procedures and/or difficulty in estimating the integrals from the ${ }^{1} \mathrm{H}$ spectrum), no appreciable difference was observed in the measured relaxation rates.

Further details of sample expression, purification, and preparation are given in the experimental section.

Relaxation Time Measurements. For each sample, we measured the following: ${ }^{1} \mathrm{H} \mathrm{R} \mathrm{R}_{1}$ from ${ }^{1} \mathrm{H}$ spectra, ${ }^{1} \mathrm{H} \mathrm{R}_{1}$ on ${ }^{13} \mathrm{C}$ through cross polarization (CP), methyl ${ }^{13} \mathrm{C} \mathrm{R}_{1}$, amide ${ }^{15} \mathrm{~N} \mathrm{R}_{1}$, and lysine ${ }^{15} \mathrm{~N}_{\zeta} \mathrm{R}_{1}$. Data were taken at $11.7 \mathrm{~T}\left(500 \mathrm{MHz}{ }^{1} \mathrm{H}\right.$ frequency) and $21.1 \mathrm{~T}$ (900 MHz ${ }^{1} \mathrm{H}$ frequency) for $\mathrm{SH} 3$ and
Sendai Ntail and only $11.7 \mathrm{~T}$ for OmpG, while the data for GB1 were taken at 9.4, 11.7, 14.1, and, $18.8 \mathrm{~T}$ and are here reproduced from ref. ${ }^{33}$ For GB1, SH3, and OmpG, data were taken over a temperature range from 100 to $280 \mathrm{~K}$. The Ntail sample melts at around $250 \mathrm{~K}$ and therefore transits to rapid overall isotropic motion, and consequently, the signals in CPexcited spectra disappear, since $\mathrm{CP}$ is not efficient in this motional regime. This sets an upper limit on temperature for Sendai Ntail for the measurements with our approach.

Figures 2-6 show the different measured $R_{1}$ rates as a function of temperature at different magnetic fields, with the

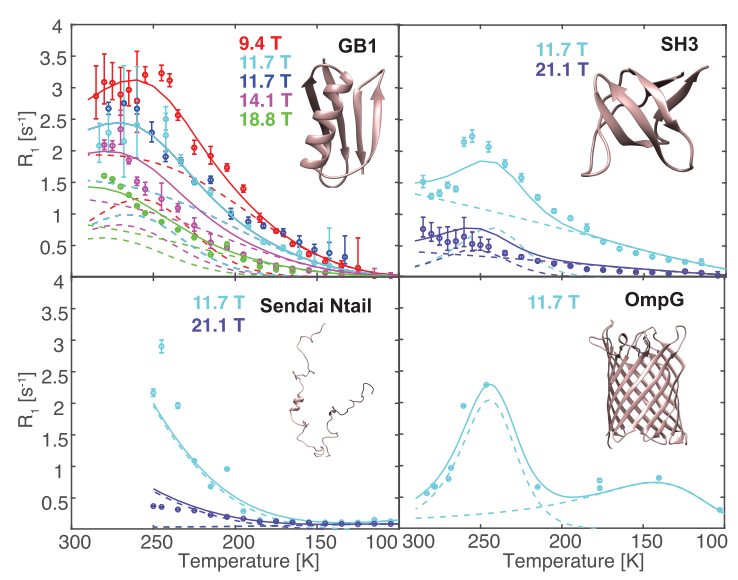

Figure 2. Measured $R_{1}$ of the directly observed proton signals, $R_{1 H}$, which is mainly associated with the solvent, together with the associated error bar, as a function of temperature. Each color corresponds to a different magnetic field, with red being $400 \mathrm{MHz}$, blue and cyan $500 \mathrm{MHz}$, purple $600 \mathrm{MHz}$, green $800 \mathrm{MHz}$, and violet $900 \mathrm{MHz}$. The solid line is the result of the joint fit for all the data, simultaneously, to a model with $N=2$ modes for GB1, SH3, and OmpG and to $N=1$ for Sendai Ntail. The dotted lines show the contribution of each mode to the full relaxation process. The number of modes needed for the fits was statistically justified with an $\mathrm{F}$ test ( $p$ $<0.05)$.

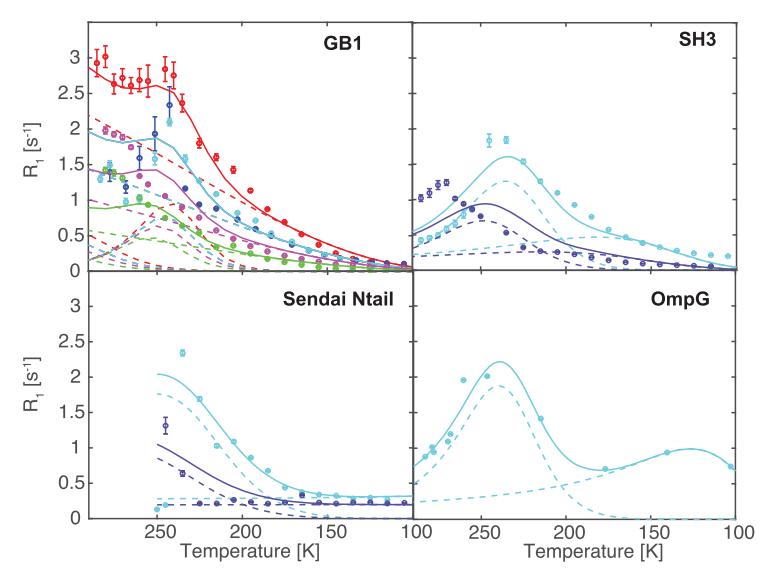

Figure 3. Measured $\mathrm{R}_{1}$ of the proton signals measured through $\mathrm{CP}$ to ${ }^{13} \mathrm{C}$ are shown as a function of temperature. The signals arise mostly from the protons bound to the protein, but since the relaxation times are in the order of magnitude of seconds, proton spin diffusion takes place. This homogenizes the relaxation rates with the crystal water. Color and line codes are the same as Figure 2. $N=2$ modes for Sendai Ntail, SH3, and OmpG; and to $N=3$ for GB1. The number of modes needed for the fits was statistically justified with an $\mathrm{F}$ test $(p<$ $0.05)$. 


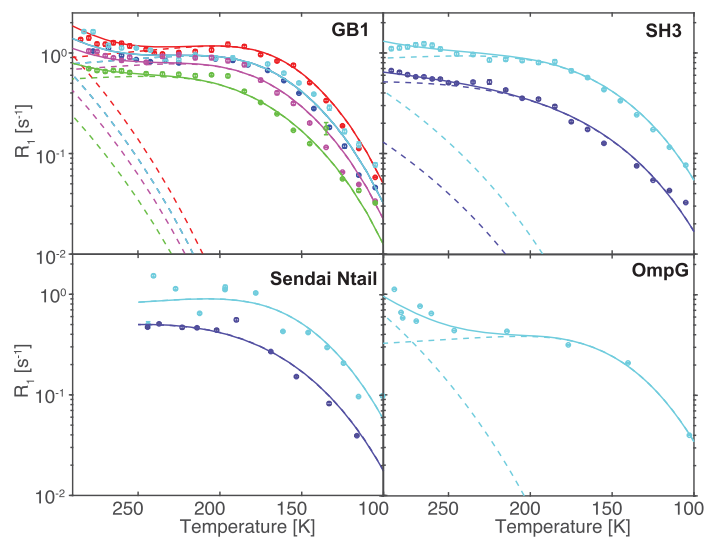

Figure 4. Measured ${ }^{13} \mathrm{C} \mathrm{R}_{1}$ of the methyl signals near $0 \mathrm{ppm}$, which are mainly associated with the hydrophobic side chains, together with the error estimated bars, as a function of temperature. Color and line codes are the same as Figure 2. $N=2$ modes for GB1, SH3, and OmpG; and $N=1$ for Sendai Ntail. The number of modes needed for the fits was statistically justified with an $\mathrm{F}$ test $(p<0.05)$.

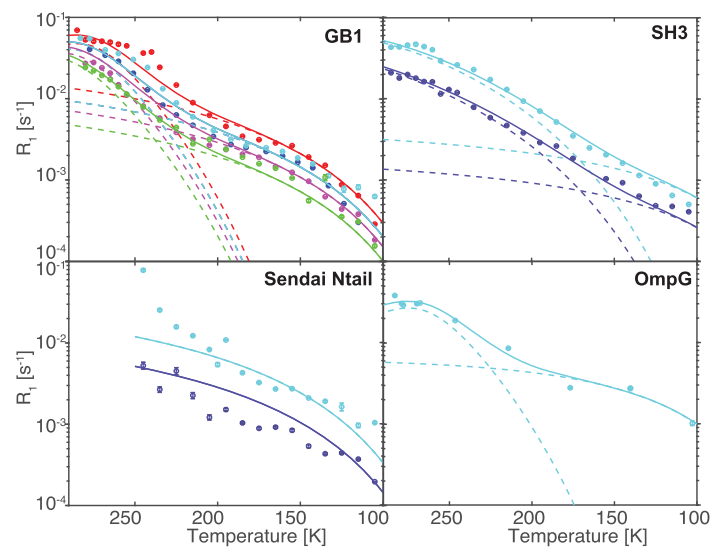

Figure 5. Measured ${ }^{15} \mathrm{~N} \mathrm{R}_{1}$ of the signals near $120 \mathrm{ppm}$, which are mainly associated with the backbone and hydration water, together with the associated estimated error bars, as a function of temperature Color and line codes are the same as Figure 2. $N=2$ modes for GB1, SH3, and OmpG; and to $N=1$ for Sendai Ntail. The number of modes needed for the fits was statistically justified with an F test ( $p<$ $0.05)$.

corresponding fits, while Figure 7 shows the result of a constrained fit (as explained below), where the energy of the second mode was fixed to $30 \mathrm{~kJ} \cdot \mathrm{mol}^{-1}$. The error bars in Figures $2-7$ are the $95 \%$ interval of certainty for the fits of $R_{1}$. More details about the extraction of $R_{1}$ values from the saturation recovery curves using exponential fits are given in the Supporting Information.

Motional Modes and Activation Energies. All curves were analyzed in terms of two motional modes except for GB1 where three modes were used for fitting ${ }^{1} \mathrm{H} \mathrm{CP}$ relaxation. We note that low temperatures lead to a loss of resolution in the NMR spectrum ${ }^{39-42}$ to the extent that site-specific information is no longer accessible, but type-specific information may still be obtained. Thus, the rates reported are the sum of contributions from all of the contributing nuclei of a given type in each protein. Here, we measure longitudinal relaxation rates, which are expected to be sensitive to picosecondnanosecond timescale motions. Each relaxation curve is fitted to extract a single $R_{1}$ value, as described in the Supporting

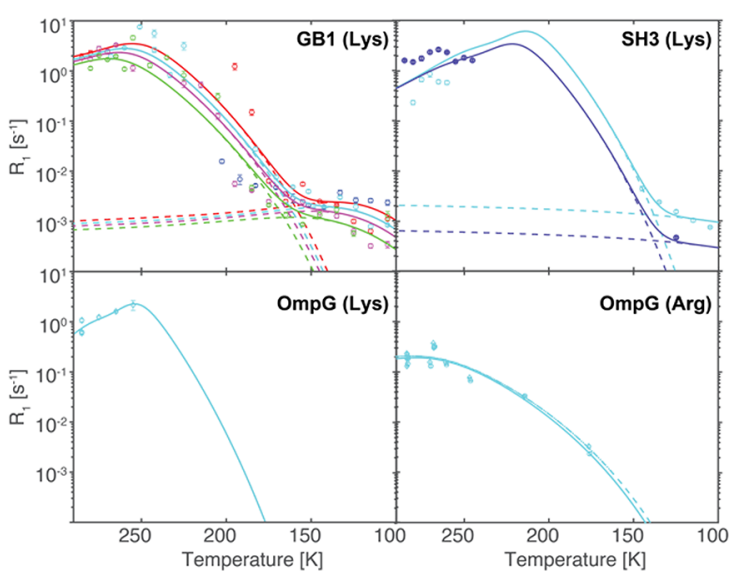

Figure 6. Measured ${ }^{15} \mathrm{~N} \mathrm{R}_{1}$ of the signals near $30 \mathrm{ppm}$ (top row), which are associated with the hydrophilic lysine side chains, together with the associated estimated error bar, as a function of temperature. The solid and dashed lines are the fits to the data, with color and line codes the same as Figure 2, and $N=2$ modes are for both GB1 and SH3. The number of modes needed for the fits was statistically justified with an $\mathrm{F}$ test $(p<0.05)$. In the bottom row, the equivalent data on lysine signals of OmpG obtained at $500 \mathrm{MHz}$ are shown to the left, and the ${ }^{15} \mathrm{~N} \mathrm{R}_{1}$ of arginine guanidinium groups of OmpG is shown to the right. Both curves represent fits with only one mode $(\mathrm{N}$ $=1$ ).

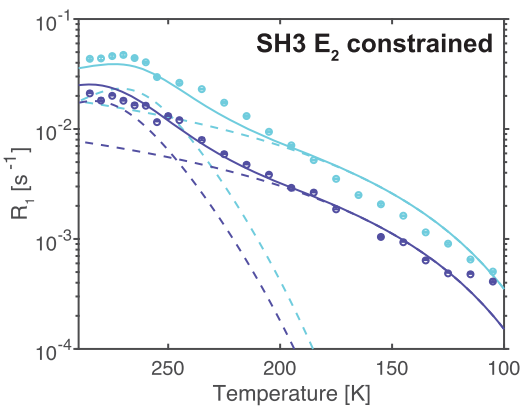

Figure 7. Measured ${ }^{15} \mathrm{~N} \mathrm{R}_{1}$ of the signals near $120 \mathrm{ppm}$ of $\mathrm{SH} 3$ previously shown in Figure 5, which are mainly associated with the backbone and hydration water, together with the associated estimated error bars, as a function of temperature. Each color corresponds to a different magnetic field, with blue, $500 \mathrm{MHz}$, and violet, $900 \mathrm{MHz}$. The solid line is the result of the joint fit for all the data, simultaneously, to a model with $N=2$ modes. The dotted lines show the contribution of each mode to the full relaxation process. The energy of the second mode was arbitrarily fixed to $30 \mathrm{~kJ} \cdot \mathrm{mol}^{-1}$ in order to better illustrate the uncertainty on the energy of the second mode.

Information, which are the values reported in Figures 2-6 (the numerical values are given in the Supporitng Information).

We use Redfield theory with thermally activated motions to describe relaxation. ${ }^{43}$ We assume that each motional mode has a temperature-dependent rate according to an Arrhenius relation: ${ }^{44}$

$$
\tau_{k}=\tau_{\infty, k} \mathrm{e}^{\left(E_{k} / R T\right)}
$$

where $\tau_{\mathrm{k}}$ is the correlation time of motion and $E_{k}$ is the activation energy. $\tau_{\infty, k}$ is analogous to a timescale and in abstract terms correspond to the correlation time of the motion at infinite temperature. Note that comparisons should be made between the times $\tau_{k}$ at given temperatures, since $\tau_{\infty, k}$ is not particularly informative. 
The correlation times are related to the longitudinal relaxation rates though spectral densities. In particular for protons, the relaxation rate induced by the dipolar coupling is given by the following expression: ${ }^{20}$

$$
R_{1 \mathrm{H}}^{D D}=C_{\text {interaction }}\left(J\left(\omega_{\mathrm{H}}\right)+4 J\left(2 \omega_{\mathrm{H}}\right)\right)
$$

where $J(\omega)$ is the spectral density (defined below) and $\omega_{\mathrm{H}}$ is the proton Larmor frequency.

The relaxation rate induced by the dipolar coupling for heteroatoms is given instead by

$$
\begin{aligned}
R_{1 \mathrm{X}}^{D D}= & C_{\text {interaction }}\left(J\left(\omega_{\mathrm{H}}-\omega_{\mathrm{X}}\right)+3 J\left(\omega_{\mathrm{X}}\right)\right. \\
& \left.+6 J\left(\omega_{\mathrm{H}}+\omega_{\mathrm{X}}\right)\right)
\end{aligned}
$$

where $\omega_{\mathrm{X}}$ is the heteroatom Larmor frequency $\left({ }^{13} \mathrm{C}\right.$ or $\left.{ }^{15} \mathrm{~N}\right)$.

Finally, the relaxation rate due to the chemical shift anisotropy (CSA) for heteroatoms is given by

$$
R_{1 \mathrm{X}}^{C S A}=C_{\text {interaction }} J\left(\omega_{\mathrm{X}}\right)
$$

Assuming a Gaussian-Markoff model for the modes results in a spectral density $J(\omega)$ at frequency $\omega$, with a sum over the different modes.

$$
J(\omega)=\sum_{k=1}^{N} C_{k, \text { amplitude }} \frac{\tau_{k}}{1+\omega^{2} \tau_{k}^{2}}
$$

with $N$ being the number of different motional modes (here 1 , 2 , or 3$). C_{k}$ amplitude is a dimensionless factor associated with the amplitude of the motion. Note that in the following, we assume that the amplitude of the motion is constant as a function of temperature, which was previously shown to be a reasonable approximation for this analysis. ${ }^{33}$ This avoids overfitting the relaxation data.

For each type of rate, the rates measured as a function of the temperature and magnetic field were jointly fit to the model given by eqs $1-5$ in order to extract the activation energies and the $\tau_{\infty, \mathrm{k}}$ prefactors. Each curve was fit with a variable number of modes, with the most significant fit being reported. For most cases, this corresponds to $N=2$ modes being required to properly fit the data. The number of modes needed for the fits was statistically justified with an $\mathrm{F}$ test $(p<0.05)$.

From this fit, we extract three different parameters, informing us about motions. The first one is a combination of $C_{k \text {, amplitude }}$ and $C_{\text {interaction }}$. As we are dealing here with an ensemble of observables, the direct interpretation of these parameters in terms of the geometry of the interaction is not straightforward. The second one is the pre-exponential factor, $\tau_{\infty, k}$, which is usually affected by imprecision and therefore is not highly informative; finally, the energy of activation is the most informative parameter obtained with this approach. It will be noted as $E_{k}$, with $k$ being the motional mode. $k=1$ is associated to the motional mode driving relaxation at a lower temperature and $k=2$ to the motional mode driving relaxation at a higher temperature.

Figure 8 shows the activation energies determined from the data of Figures 2-7. The numerical values and the prefactors are given in the Supporting Information.

In order to estimate the errors on the extracted constants, a Monte Carlo procedure using 500 iterations, with similar displacements from the fitted curve to those observed experimentally, was performed. The error bars in Figure 8 represent the $95 \%$ uncertainty obtained from the Monte Carlo
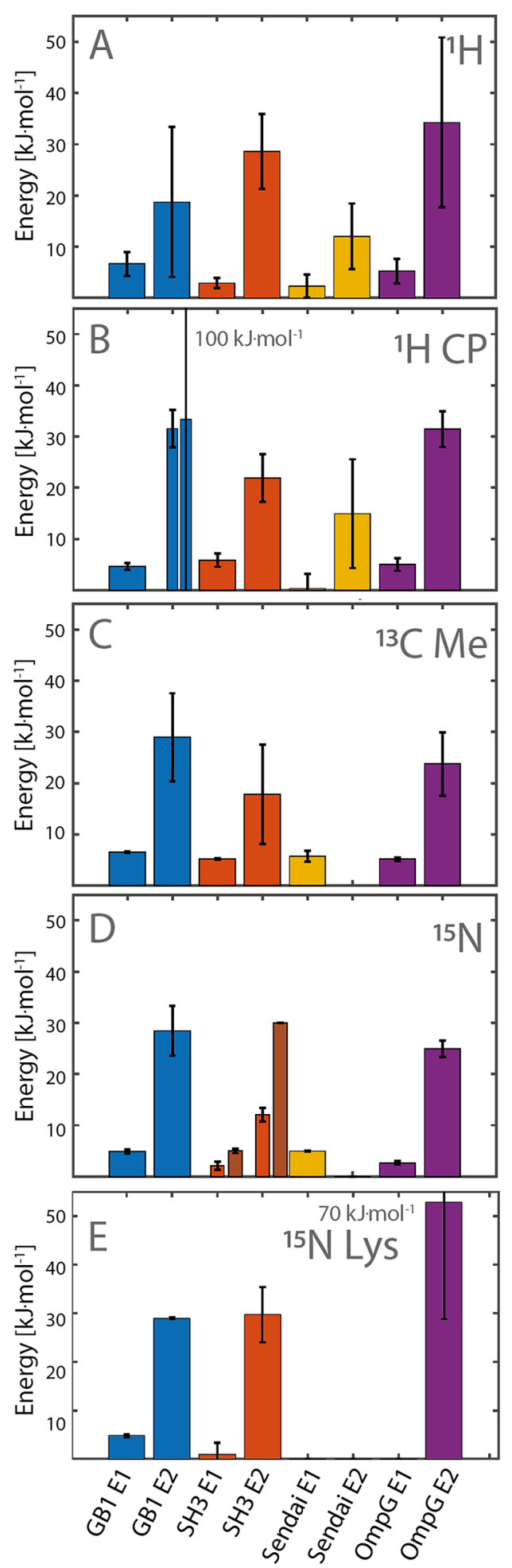

Figure 8. Energies extracted from the fits to the different relaxation rates for the dynamics associated with the five probes. Error bars are estimated based on a Monte Carlo analysis as described in the Motional Mode and Activation Energies subsection. The double bars in the ${ }^{1} \mathrm{H}$ CP panel represent the modes 2 and 3 . The darker bars in the ${ }^{15} \mathrm{~N}$ panel for the $\mathrm{SH} 3$ resonances represent the data for the forced fit shown in Figure 7.

procedure. Note that this method measures the precision and not the accuracy of the fits. 


\section{DISCUSSION}

Relaxation Rates. We first briefly recall what each of the measured rates reports on. ${ }^{1} \mathrm{H} \mathrm{R}_{1}$ are evaluated by integrating the whole ${ }^{1} \mathrm{H}$ spectrum (Table S1). As the spectrum is largely dominated by the solvent (and lipid for OmpG) signal, the measured $\mathrm{R}_{1}$ mainly reports on the solvent dynamics (Figure 2).

${ }^{1} \mathrm{H} \mathrm{R}_{1}$ through ${ }^{13} \mathrm{C} \mathrm{CP}$ are evaluated from full integration of the ${ }^{13} \mathrm{C}$ CP spectrum, and the measured rates are shown in Figure 3 . The $\mathrm{CP}$ signals arise mostly from the protons bound to the protein, but since the relaxation rates are on the second timescale, ${ }^{1} \mathrm{H}-{ }^{1} \mathrm{H}$ spin diffusion will average relaxation rates between hydration water, channel water in the microcrystal, and any bulk water by diffusion length that scales up to around hundreds of nanometers from the protein surface even at intermediate temperatures. ${ }^{45}$ Therefore, the ${ }^{1} \mathrm{H}$ relaxation observed through the ${ }^{13} \mathrm{C}$ spectra probes a weighted average of near and far protons. If this is the case, the measured relaxation rates are seen to be uniform at the protein scale, i.e., across all the ${ }^{13} \mathrm{C}$ chemical shifts (see Figure S2). Some typical spectra are shown in Figure S4.

Methyl ${ }^{13} \mathrm{C} \mathrm{R}_{1}$ is mostly associated with aliphatic side chains (Figure 4). Amide ${ }^{15} \mathrm{~N} \mathrm{R}_{1}$ is associated with the protein backbone and hydration water (Figure 5). Lysine ${ }^{15} \mathrm{~N} \mathrm{R}_{1}$ probes hydrophilic side chains (Figure 6).

At first sight, the most obvious difference in the behavior between samples in the raw relaxation data shown in Figures $2-6$ is for the Sendai Ntail, which, since it is a frozen solution, melts to an isotropic liquid above $250 \mathrm{~K}$ (which is the highest temperature at which CP-based experiments work). A side effect is also that the isotropic motion will average dipolar couplings to zero, and the cross-polarization experiments will no longer work. Since the other proteins are precipitated in lipid bilayers (OmpG) or are microcrystalline ( $\mathrm{SH} 3$ and GB1), they remain solid even when the bulk solvent unfreezes. As a result, in those samples, overall tumbling is still highly restricted, ${ }^{7}$ and cross polarization is still effective.

The characteristic relaxation behavior that is observed for the other proteins above $250 \mathrm{~K}$ is therefore not observable in Sendai Ntail. This has the consequence that for the heteronuclear relaxation rates, only the low energy mode is measurable in Sendai Ntail. However, this does not in itself mean that the underlying dynamics up to the melting point are different.

The other obvious difference is in the data for ${ }^{15} \mathrm{~N}$ lysine $\mathrm{R}_{1}$, shown in Figure 6. Here, we observe a loss of signal for GB1 and $\mathrm{SH} 3$ between the two measurement points 150 and $250 \mathrm{~K}$. This has been associated with intermediate exchange motions interfering with decoupling or MAS (motions that are much slower than the motions impacting $\left.\mathrm{R}_{1}\right){ }^{20,46}$ In the case of Sendai Ntail it was not possible to properly integrate the signal of interest due to the low signal-to-noise ratio, and the analysis is thus omitted. The case of OmpG is particularly interesting, since the data obtained here can potentially provide mechanistic insight into the function of positively charged residues in the context of the positive-inside rule determining the orientation of helical membrane proteins. Although we are investigating a $\beta$-barrel protein, the interaction of the positively charged head groups of arginine and lysine with negatively charged groups of the lipids, mainly phosphates, may lead to altered relaxation behavior in comparison to the corresponding residues in the three soluble proteins. OmpG contains six lysine residues, of which five are situated indeed in the loops, turns, or just at the ends of the $\beta$-strands that may readily interact with lipid molecules, and fifteen arginine residues, with the majority of them located in loops or turns but also several pointing into the pore. We have included the corresponding curves in the lower row of Figure 6. The lysine side chain ${ }^{15} \mathrm{~N}$ signals are observable only in the high temperature range (in our measurements above $250 \mathrm{~K}$ ), and they do not reappear in the low temperature range. This seems to already illustrate a different behavior for these slow motions in the water-soluble proteins as compared to the membrane protein. The data here might suggest that the slow lysine side chain motions are significantly modified in OmpG, with an earlier onset in the membrane protein as compared to the water-soluble proteins, even if caution must be used here as the number of experimental points is low. Example ${ }^{15} \mathrm{~N}$ spectra illustrating this intermediate exchange effect are shown in Figure S5, including spectra with high signal-to-noise ratios, to confirm that the OmpG signals do not reappear at low temperatures. Similarly, the arginine side chain ${ }^{15} \mathrm{~N}$ signals are too small at low temperatures to determine $\mathrm{R}_{1}$ reliably (Figure 6).

If we look in detail at the ${ }^{13} \mathrm{C}$-filtered ${ }^{1} \mathrm{H}$ relaxation data shown in Figure 3, which is sensitive to the protons of the protein, the hydration shell, and a contribution from the solvent outside the hydration shell, ${ }^{35}$ we find that three modes can be distinguished in case of the richer GB1 dataset, but this was not the case for the other proteins that only required two modes to explain the data. However, the two high-energy modes found for GB1 are very similar in energy, and we consider them as one mode in the following comparison.

Figure 7 is a fit of the $\mathrm{SH} 3{ }^{15} \mathrm{~N}$ backbone signal (like Figure 5) with the high-energy mode fixed at $30 \mathrm{~kJ} \cdot \mathrm{mol}^{-1}$. This illustrates the relatively low accuracy of the previous fit and illustrates that in this case, the difference between GB1 and $\mathrm{SH} 3$ illustrated in Figure 8 might not be significant, as forcing the energy to be similar also yields a statistically acceptable fit. We note that for other cases, forcing energies to be similar results in statistically unacceptable fits, as discussed previously. ${ }^{33}$

Activation Energies. We are now in a position to compare the energetics of the fast motion modes exhibited by the different types of protein, as shown in Figure 8.

Before discussing the results in detail, it is important to note that we do not obtain a single activation energy for each mode per residue type. Our description is obviously an approximation, because the relaxation rates measured are averaged over many sites and thus is due to a distribution of motions over a range of timescales and amplitudes. The activation energies and amplitudes obtained are thus weighted averages. Nonetheless, this implies both that if we can identify two modes with statistical significance, then these modes are measurably different and that any differences in these averages between proteins correspond to significant changes in dynamics between proteins.

Overall, we see that qualitatively all the proteins have lowand high-energy modes in broadly similar ranges, and that this tends to confirm the idea that protein dynamics is to some extent universal. The low-energy modes range from 2 to $6 \mathrm{~kJ}$. $\mathrm{mol}^{-1}$ (excluding the Sendai Ntail mode for the protons near the protein), and the high-energy modes range from 10 to 35 $\mathrm{kJ} \cdot \mathrm{mol}^{-1}$. 
When we take a more detailed look at the activation energies, we can identify differences in behavior that can be rationalized in terms of the atomic-level structures.

The activation energies obtained from the directly measured ${ }^{1} \mathrm{H}$ rates, which probe activation energies of solvent motion both within the crystal lattices and in bulk excess water or lipid, are reported in Figure 8A. The low-energy mode is mainly due to libration of the solvent since at this temperature, water rotation as well as translation is almost entirely blocked. ${ }^{47}$ The high-energy mode has been assigned to solvent rotations. We observe quite consistent behavior across the series, with the most striking difference being the relatively low value for the high-energy process in the Sendai Ntail sample. We may attribute this in part to the less stringent requirement for solvent ordering around the intrinsically disordered protein as compared to the more ordered organization of the solvent in the other three, somewhat compartmentalized samples, even if this should be considered speculative, due to the low number of experimental points at the high temperature for the Sendai Ntail. We assume that the latter is particularly the case for the samples containing both the crystalline GB1 and $\mathrm{SH} 3$ structures due to the presence of spaces in the crystal lattice and also for the one with the membrane-inserted OmpG where the extended lipid bilayer-water interface is expected to contribute dominantly to this effect rather than the protein. Indeed, the relaxation behavior of the overall ${ }^{1} \mathrm{H}$ signal of the OmpG sample indicates the highest activation energy of the set for these motions, whereby it should be noted that part of the observed $R_{1}$ rate is obtained on the signals of the lipid bilayer.

The measured activation energies obtained from the ${ }^{13} \mathrm{C}$ filtered ${ }^{1} \mathrm{H}$ relaxation rates, which are sensitive to dynamics of protons in the solvent close to the protein, are shown in Figure $8 \mathrm{~B}$. The low-energy mode has been attributed to fluctuation of side chains within local energy wells, as well as to the abovementioned restricted solvent motions. The high-energy mode is assigned to solvent rotation and larger amplitude side chain motions, with the first being concomitant with the second. ${ }^{47}$ The most obvious difference is the energy for the first mode of the very dilute and the most highly water accessible Sendai Ntail being one order of magnitude lower than its counterparts. We can also notice that the more complete data set of GB1 allow us to distinguish three modes here. We previously noted ${ }^{20,33}$ that the ${ }^{1} \mathrm{H}$ and ${ }^{13} \mathrm{C}$-filtered ${ }^{1} \mathrm{H}$ signals appear to share similar temperature dependence of $\mathrm{R}_{1}$ as the backbone relaxation, and it is not possible to distinguish between the solvent-inducing (slaving) protein motions or vice versa. The solvation water moves in concert with the protein. Therefore in this case, the ${ }^{13} \mathrm{C}$-filtered ${ }^{1} \mathrm{H}$ relaxation reports on the slow component of the backbone motion of Sendai Ntail. We note that the slow backbone motion is invisible in the ${ }^{15} \mathrm{~N}$ relaxation but measurable here and is, not surprisingly, lower than for the other proteins. This yields activation energies that are very similar to those measured in solution. ${ }^{48}$ This is coherent with previous descriptions in solution, ${ }^{32}$ although they have been made at a higher temperature.

Measures of ${ }^{13} \mathrm{C}$ relaxation in methyl groups report on the activation energies of the methyl group motions in the protein and are reported in Figure 8C. The low-energy mode was assigned to methyl rotation, while the higher mode was associated with the jump between different side-chain rotameric states. ${ }^{20}$ The first observation is that the low-energy mode (i.e., rotation) seems to be extremely stable between the four proteins. This might be unexpected, since methyl rotations are known to vary significantly. ${ }^{49,50}$ However, it is not surprising that there is overall very high similarity here, due to the fact that we measure the average activation energy for all the methyl groups in the protein. The average environment of methyl groups in the proteins is expected to be similar, and therefore the average activation energies should be similar among proteins. It is also known that methyl group rotation does not depend strongly on the solvent, such that the contact with lipids for OmpG is not expected to significantly affect the dynamics. 51

Measures of ${ }^{15} \mathrm{~N}$ amide relaxation rates, which are mostly influenced by backbone motions, are shown in Figure 8D. We note that amide bonds also interact with water, depending on the fold. The low-energy mode was assigned to small scale and small amplitude motions, while the high-energy mode was assigned to collective small amplitude backbone motions, for example, of the peptide plane. ${ }^{20}$ The main concern here, as mentioned above, is with the SH3 fit that is not highly accurate. The forced fit shown in Figure 7 is an indication that the differences between $\mathrm{SH} 3$ and GB1 might not be significant, especially if we notice that the energies are similar among the other proteins as shown in Figure 8. If we discount this difference, then these activated processes seem to be very similar between the four proteins.

The ${ }^{15} \mathrm{~N}$ relaxation rates of the arginine guanidinium group and the lysine $\mathrm{NH}_{3}{ }^{+}$are sensitive to motions related to the hydrophilic side chains enabling interesting comparisons, although the data set is somewhat incomplete. Arginines are absent from GB1, and data for $\mathrm{SH} 3$ are missing at the high temperature due to low signal-to-noise ratios and chemical exchange, and therefore only the low-energy motions are observable. The energies extracted from the arginine ${ }^{15} \mathrm{~N} \mathrm{R}_{1}$ for SH3 and Sendai Ntail are shown in Figure S6.

The analysis of the observable lysine ${ }^{15} \mathrm{~N} \mathrm{R}_{1} \mathrm{~s}$ for GB1, SH3, and OmpG yields the energies shown in Figure $8 \mathrm{E}$. The data again indicate at least a bimodal distribution of energies for side-chain motions in GB1 and SH3. It appears that the difference is the most significant in the comparison between OmpG and the other proteins, where we observe a higher activation energy associated with the second mode in OmpG. This might be associated with higher rigidity of the lysine side chains in the loops and turns. In OmpG, they are all available for interactions with negatively charged lipids in the membrane, in particular phosphatidylglycerol. The corresponding activation energies associated with the arginine guanidium group signals (Figure S6) cannot be compared to those of $\mathrm{SH} 3$ and Sendai Ntail, but they are closer to the values observed for backbone amide nitrogen. This implies the mixture of rigidity and flexibility that is represented by the loop/ $\beta$-sheet composition of OmpG: the larger part of the arginine residues in OmpG may interact with the membrane, but a large fraction does not. As discussed above, due to the disappearance/low intensity of side-chain ${ }^{15} \mathrm{~N}$ signals below $250 \mathrm{~K}$, it was not possible to extract the motional parameters associated with the low-energy mode of lysine residues in OmpG. This difference also corroborates an overall difference in the lysine side-chain dynamics. We speculate therefore that the data reflect interactions of the positively charged lysine side chains with the negatively charged phosphate groups of the lipids in the bilayer, next to their hydrophobic interactions. 


\section{CONCLUSIONS}

In conclusion, we have shown that by measuring a series of six relaxation rates at variable temperatures and variable magnetic fields, we can obtain averaged activation energies for motions in different parts of a series of four proteins representing three of the broad classes of proteins: soluble globular proteins, intrinsically disordered proteins, and membrane proteins.

While these activation barriers are measured with experiments at cryogenic temperatures, it is important to note that knowledge of these fundamental parameters allows us to predict from the resulting Boltzmann factors the dynamic behavior of the proteins at physiological temperatures.

We observe many similarities, notably in backbone motions and methyl group rotations, suggesting that proteins do share universal dynamic features. For these motions, similar activation energies are measured for all four proteins.

Beside this, some differences are evident, with the main observed difference between the various types of protein occurring at the interface between the solvent and protein. This is unsurprising since the influence of the solvent on the dynamics of proteins has been well documented. ${ }^{9,10,21,46,52,53}$

One difference is related to the reduced activation energy of the Sendai Ntail (reported on by ${ }^{1} \mathrm{H}$ and ${ }^{13} \mathrm{C}$-mediated ${ }^{1} \mathrm{H}$ relaxation). This suggests that the well-defined energy minimum associated with a folded protein impacts local dynamic modes by constraining the degrees of conformational freedom, while this does not happen in IDPs because the energy landscape is flatter. This lack of restraint imposed by the rigid scaffold appears to lead to lower activation energies. In the case of the membrane protein, the activiation energy is similar to the water-soluble proteins, suggesting that the fold is more important than the nature of the solvent.

Another difference is observed in the lysine side chains of the membrane protein for both ${ }^{15} \mathrm{~N} \mathrm{R}_{1}$ and the slow exchange behavior, pointing to an overall difference in lysine motions between the membrane protein and the microcrystalline globular proteins. This is consistent with interactions of the positively charged lysine head group with negatively charged lipids in the bilayer and suggests a possible mechanism for the role of positively charged amino acids in membrane protein biology. The comparison of the lysine data obtained on $\mathrm{SH} 3$ and GB1 with the activation energy $E^{2}$ obtained for OmpG also suggests a stronger interaction with the membrane than with the aqueous environment within the crystallites present in the SH3 and GB1 samples.

One of the main limitations with the approach used here is the lack of site-specific resolution, due mostly to signal overlap and large inhomogeneous broadening at low temperatures.

In this respect, it is interesting to note that the two dynamic modes become activated at temperatures higher than those usually used in structural studies performed by DNP NMR and cryo electron microscopy (cryoEM), confirming that these techniques observe ensembles of frozen proteins, and this contributes to the limits of resolution attainable with these methods.

In DNP NMR, the spectra are dominated by inhomogeneous signal broadening at very low temperatures due slow interconversion between/of different conformers, as has been discussed in detail elsewhere. ${ }^{20,54}$ Even if we did not investigate the individual contributions of homogenous and inhomogeneous broadening to the observed linewidths in the present case, the observed broadening for the range of proteins analyzed here is compatible with inhomogeneous broadening being dominant. In cryoEM, the image is therefore reconstructed from snapshots of the different conformers present.

The most obvious future development in that sense to measure dynamics would be to perform site-specific labeling to access site-specific dynamics, potentially allowing identification of local differences in the protein dynamic landscape.

Other future work will be directed to extending this analysis to more proteins, in order to validate the preliminary observations here and further refine the understanding of the similarities and differences between dynamics in proteins.

\section{ASSOCIATED CONTENT}

\section{Supporting Information}

The Supporting Information is available free of charge at https://pubs.acs.org/doi/10.1021/acs.jpcb.0c10188.

Further experimental details, NMR spectra, details of the fits (PDF)

Signal intensities for all relaxation rates (ZIP)

\section{AUTHOR INFORMATION}

\section{Corresponding Author}

Lyndon Emsley - Institut des Sciences et Ingénierie Chimiques, Ecole Polytechnique Fédérale de Lausanne (EPFL), 1015

Lausanne, Switzerland; (1) orcid.org/0000-0003-13602572; Email: lyndon.emsley@epfl.ch

\section{Authors}

Baptiste Busi - Institut des Sciences et Ingénierie Chimiques, Ecole Polytechnique Fédérale de Lausanne (EPFL), 1015 Lausanne, Switzerland

Jayasubba Reddy Yarava - Leibniz-Forschungsinstitut für Molekulare Pharmakologie, 13125 Berlin, Germany; Freie Universität Berlin, 14195 Berlin, Germany

Andrea Bertarello - Institut des Sciences et Ingénierie Chimiques, Ecole Polytechnique Fédérale de Lausanne (EPFL), 1015 Lausanne, Switzerland; @ orcid.org/00000003-3705-1760

François Freymond - Institut des Sciences et Ingénierie Chimiques, Ecole Polytechnique Fédérale de Lausanne (EPFL), 1015 Lausanne, Switzerland

Wiktor Adamski - Université Grenoble Alpes, CNRS, CEA, IBS, 38000 Grenoble, France

Damien Maurin - Université Grenoble Alpes, CNRS, CEA, IBS, 38000 Grenoble, France

Matthias Hiller - Leibniz-Forschungsinstitut für Molekulare Pharmakologie, 13125 Berlin, Germany; Freie Universität Berlin, 14195 Berlin, Germany

Hartmut Oschkinat - Leibniz-Forschungsinstitut für Molekulare Pharmakologie, 13125 Berlin, Germany; Freie Universität Berlin, 14195 Berlin, Germany; 이이.org/ 0000-0002-4384-9544

Martin Blackledge - Université Grenoble Alpes, CNRS, CEA, IBS, 38000 Grenoble, France; 이이이.org/0000-00030935-721X

Complete contact information is available at:

https://pubs.acs.org/10.1021/acs.jpcb.0c10188

\section{Notes}

The authors declare no competing financial interest. 
The NMR raw data are available from http://dx.doi.org/10. 5281/zenodo.4542887. Data are made available under the license CC-BY-4.0 (Creative Commons Attribution-ShareAlike 4.0 International).

\section{ACKNOWLEDGMENTS}

We are grateful for financial support from the Swiss National Science Foundation grant no. 200021_162514 and the Swiss National Centre of Competence in Research (NCCR) Chemical Biology.

\section{REFERENCES}

(1) Rasmussen, B. F.; Stock, A. M.; Ringe, D.; Petsko, G. A. Crystalline ribonuclease A loses function below the dynamical transition at 220 K. Nature 1992, 357, 423-424.

(2) Anderson, A. C. The process of structure-based drug design. Chem. Biol. 2003, 10, 787-797.

(3) Henzler-Wildman, K. A.; Lei, M.; Thai, V.; Kerns, S. J.; Karplus, M.; Kern, D. A hierarchy of timescales in protein dynamics is linked to enzyme catalysis. Nature 2007, 450, 913-916.

(4) Khodadadi, S.; Sokolov, A. P. Atomistic details of protein dynamics and the role of hydration water. Biochim. Biophys. Acta Gen. Subj. 2017, 1861, 3546-3552.

(5) Buck, M.; Boyd, J.; Redfield, C.; MacKenzie, D. A.; Jeenes, D. J.; Archer, D. B.; Dobson, C. M. Structural determinants of protein dynamics: analysis of ${ }^{15} \mathrm{~N}$ NMR relaxation measurements for mainchain and side-chain nuclei of hen egg white lysozyme. Biochemistry 1995, 34, 4041-4055.

(6) Fenimore, P. W.; Frauenfelder, H.; Magazu, S.; McMahon, B. H.; Mezei, F.; Migliardo, F.; Young, R. D.; Stroe, I. Concepts and problems in protein dynamics. Chem. Phys. 2013, 424, 2-6.

(7) Lamley, J. M.; Lewandowski, J. R. Relaxation-based magic-angle spinning NMR approaches for studying protein dynamics. Emagres 2016, 5, 1423-1434.

(8) Jansson, H.; Swenson, J. The protein glass transition as measured by dielectric spectroscopy and differential scanning calorimetry. Biochim. Biophys. Acta 2010, 1804, 20-26.

(9) Jansson, H.; Bergman, R.; Swenson, J. Role of solvent for the dynamics and the glass transition of proteins. J. Phys. Chem. B 2011, 115, 4099-4109.

(10) Lipps, F.; Levy, S.; Markelz, A. G. Hydration and temperature interdependence of protein picosecond dynamics. Phys. Chem. Chem. Phys. 2012, 14, 6375-6381.

(11) Knab, J. R.; Chen, J.-Y.; He, Y.; Markelz, A. G. Terahertz measurements of protein relaxational dynamics. Proc. IEEE 2007, 95 , $1605-1610$.

(12) Frauenfelder, H.; Chen, G.; Berendzen, J.; Fenimore, P. W.; Jansson, H.; McMahon, B. H.; Stroe, I. R.; Swenson, J.; Young, R. D. A unified model of protein dynamics. Proc. Natl. Acad. Sci. U. S. A. 2009, 106, 5129-5134.

(13) Parak, F.; Formanek, H. Untersuchung des Schwingungsanteils und des Kristallgitterfehleranteils des Temperaturfaktors in Myoglobin durch Vergleich von Mössbauer-Absorptionsmessungen mit Röntgenstrukturdaten. Acta Crystallogr. A 1971, 27, 573-578.

(14) Roy, R.; Hohng, S.; Ha, T. A practical guide to single-molecule FRET. Nat. Methods 2008, 5, 507-516.

(15) Weik, M.; Colletier, J. P. Temperature-dependent macromolecular X-ray crystallography. Acta Crystallogr. D 2010, 66, 437446.

(16) Tilton, R. F., Jr.; Dewan, J. C.; Petsko, G. A. Effects of temperature on protein structure and dynamics: X-ray crystallographic studies of the protein ribonuclease-A at nine different temperatures from 98 to 320K. Biochemistry 1992, 31, 2469-2481.

(17) Gaspar, A. M.; Appavou, M. S.; Busch, S.; Unruh, T.; Doster, W. Dynamics of well-folded and natively disordered proteins in solution: a time-of-flight neutron scattering study. Eur. Biophys. J. 2008, 37, 573-582.
(18) Doster, W.; Bachleitner, A.; Dunau, R.; Hiebl, M.; Lüscher, E. Thermal-properties of water in myoglobin crystals and solutions at subzero temperatures. Biophys. J. 1986, 50, 213-219.

(19) Doster, W.; Cusack, S.; Petry, W. Dynamical transition of myoglobin revealed by inelastic neutron scattering. Nature 1989, 337, 754-756.

(20) Lewandowski, J. R.; Halse, M. E.; Blackledge, M.; Emsley, L. Direct observation of hierarchical protein dynamics. Science 2015, 348, 578-581.

(21) Adamski, W.; Salvi, N.; Maurin, D.; Magnat, J.; Milles, S.; Jensen, M. R.; Abyzov, A.; Moreau, C. J.; Blackledge, M. A unified description of intrinsically disordered protein dynamics under physiological conditions using NMR spectroscopy. J. Am. Chem. Soc. 2019, 141, 17817-17829.

(22) Rovó, P.; Linser, R. Microsecond timescale protein dynamics: a combined solid-state NMR approach. ChemPhysChem 2018, 19, 3439.

(23) Schanda, P.; Ernst, M. Studying dynamics by magic-angle spinning solid-state NMR spectroscopy: principles and applications to biomolecules. Prog. Nucl. Magn. Reson. Spectrosc. 2016, 96, 1-46.

(24) Doster, W. The dynamical transition of proteins, concepts and misconceptions. Eur. Biophys. J. 2008, 37, 591-602.

(25) Frauenfelder, H.; Young, R. D.; Fenimore, P. W. Dynamics and the free-energy landscape of proteins, explored with the Mössbauer Effect and Quasi-Elastic Neutron Scattering. J. Phys. Chem. B 2013, 117, 13301-13307.

(26) Idiyatullin, D.; Nesmelova, I.; Daragan, V. A.; Mayo, K. H. Heat capacities and a snapshot of the energy landscape in protein GB1 from the pre-denaturation temperature dependence of backbone $\mathrm{NH}$ nanosecond fluctuations. J. Mol. Biol. 2003, 325, 149-162.

(27) Teeter, M. M.; Yamano, A.; Stec, B.; Mohanty, U. On the nature of a glassy state of matter in a hydrated protein: relation to protein function. Proc. Natl. Acad. Sci. U. S. A. 2001, 98, 1124211247.

(28) Vugmeyster, L.; Ostrovsky, D.; Ford, J. J.; Lipton, A. S. Freezing of dynamics of a methyl group in a protein hydrophobic core at cryogenic temperatures by deuteron NMR spectroscopy. J. Am. Chem. Soc. 2010, 132, 4038-4039.

(29) Gauto, D. F.; Hessel, A.; Rovó, P.; Kurauskas, V.; Linser, R.; Schanda, P. Protein conformational dynamics studied by ${ }^{15} \mathrm{~N}$ and ${ }^{1} \mathrm{H}$ $\mathrm{R}_{1 \rho}$ relaxation dispersion: application to wild-type and G53A ubiquitin crystals. Solid State Nucl. Magn. Reson. 2017, 87, 86-95.

(30) Yang, D. Probing protein side chain dynamics via ${ }^{13} \mathrm{C}$ NMR relaxation. Protein Pept. Lett. 2011, 18, 380-395.

(31) Mittermaier, A.; Kay, L. E. New tools provide new insights in NMR studies of protein dynamics. Science 2006, 312, 224-228.

(32) Abyzov, A.; Salvi, N.; Schneider, R.; Maurin, D.; Ruigrok, R. W.; Jensen, M. R.; Blackledge, M. Identification of dynamic modes in an intrinsically disordered protein using temperature-dependent NMR relaxation. J. Am. Chem. Soc. 2016, 138, 6240-6251.

(33) Busi, B.; Yarava, J. R.; Hofstetter, A.; Salvi, N.; Cala-De Paepe, D.; Lewandowski, J. R.; Blackledge, M.; Emsley, L. Probing protein dynamics using multifield variable temperature NMR relaxation and molecular dynamics simulation. J. Phys. Chem. B 2018, 122, 96979702.

(34) Franks, W. T.; Zhou, D. H.; Wylie, B. J.; Money, B. G.; Graesser, D. T.; Frericks, H. L.; Sahota, G.; Rienstra, C. M. MagicAngle Spinning Solid-State NMR Spectroscopy of the $\beta 1$ Immunoglobulin Binding Domain of Protein G (GB1): ${ }^{15} \mathrm{~N}$ and ${ }^{13} \mathrm{C}$ Chemical Shift Assignments and Conformational Analysis. J. Am. Chem. Soc. 2005, 127, 12291-12305.

(35) Böckmann, A.; Gardiennet, C.; Verel, R.; Hunkeler, A.; Loquet, A.; Pintacuda, G.; Emsley, L.; Meier, B. H.; Lesage, A. Characterization of different water pools in solid-state NMR protein samples. J. Biomol. NMR 2009, 45, 319-327.

(36) Castellani, F.; van Rossum, B.; Diehl, A.; Schubert, M.; Rehbein, K.; Oschkinat, H. Structure of a protein determined by solid-state magic-angle-spinning NMR spectroscopy. Nature 2002, 420, 98-102. 
(37) Houben, K.; Marion, D.; Tarbouriech, N.; Ruigrok, R. W.; Blanchard, L. Interaction of the C-terminal domains of sendai virus $\mathrm{N}$ and $\mathrm{P}$ proteins: comparison of polymerase-nucleocapsid interactions within the paramyxovirus family. J. Virol. 2007, 81, 6807-6816.

(38) Hiller, M.; Krabben, L.; Vinothkumar, K. R.; Castellani, F.; van Rossum, B. J.; Kühlbrandt, W.; Oschkinat, H. Solid-state magic-angle spinning NMR of outer-membrane protein G from Escherichia coli. ChemBioChem 2005, 6, 1679-1684.

(39) Tycko, R. NMR at low and ultralow temperatures. Acc. Chem. Res. 2013, 46, 1923-1932.

(40) Jacso, T.; Franks, W. T.; Rose, H.; Fink, U.; Broecker, J.; Keller, S.; Oschkinat, H.; Reif, B. Characterization of membrane proteins in isolated native cellular membranes by dynamic nuclear polarization solid-state NMR spectroscopy without purification and reconstitution. Angew. Chem., Int. Ed. 2012, 51, 432-435.

(41) Ni, Q. Z.; Markhasin, E.; Can, T. V.; Corzilius, B.; Tan, K. O.; Barnes, A. B.; Daviso, E.; Su, Y.; Herzfeld, J.; Griffin, R. G. Peptide and protein dynamics and low-temperature/DNP magic angle spinning NMR. J. Phys. Chem. B 2017, 121, 4997-5006.

(42) Debelouchina, G. T.; Bayro, M. J.; Fitzpatrick, A. W.; Ladizhansky, V.; Colvin, M. T.; Caporini, M. A.; Jaroniec, C. P.; Bajaj, V. S.; Rosay, M.; Macphee, C. E.; et al. Higher order amyloid fibril structure by MAS NMR and DNP spectroscopy. J. Am. Chem. Soc. 2013, 135, 19237-19247.

(43) Redfield, A. G., Relaxation theory: Density matrix formulation. In Encyclopedia of Magnetic Resonance, John Wiley \& Sons, Ltd: 2007.

(44) Beckmann, P. A.; Buser, C. A.; Mallory, C. W.; Mallory, F. B.; Mosher, J. Methyl reorientation in solid 3-ethylchrysene and 3isopropylchrysene. Solid State Nucl. Magn. Reson. 1998, 12, 251-256.

(45) Schmidt-Rohr, K.; Spiess, H. W., Chapter Seven - Polymer dynamics: multidimensional exchange experiments. In Multidimensional Solid-State NMR and Polymers; Schmidt-Rohr, K.; Spiess, H. W., Eds.; Academic Press: San Diego, 1994; pp. 236-276.

(46) Siemer, A. B.; Huang, K. Y.; McDermott, A. E. Protein linewidth and solvent dynamics in frozen solution NMR. PLoS One 2012, 7, No. e47242.

(47) Lee, A. L.; Wand, A. J. Microscopic origins of entropy, heat capacity and the glass transition in proteins. Nature 2001, 411, 501504.

(48) Taricska, N.; Bokor, M.; Menyhárd, D. K.; Tompa, K.; Perczel, A. Hydration shell differentiates folded and disordered states of a Trpcage miniprotein, allowing characterization of structural heterogeneity by wide-line NMR measurements. Sci. Rep. 2019, 9, 2947.

(49) Carbonell, P.; Sol, A. D. Methyl side-chain dynamics prediction based on protein structure. Bioinformatics 2009, 25, 2552-2558.

(50) Chatfield, D. C.; Augsten, A.; D'Cunha, C. Correlation times and adiabatic barriers for methyl rotation in SNase. J. Biomol. NMR 2004, 29, 377-385.

(51) A ${ }^{1} \mathrm{H}$ nuclear magnetic relaxation study of the mechanism and solvent dependence of the motion of predominantly syndiotactic poly(methyl methacrylate) in dilute solution. Polymer 1980, 21, 381387, DOI: 10.1016/0032-3861(80)90006-3.

(52) Fenwick, R. B.; Oyen, D.; Dyson, H. J.; Wright, P. E. Slow dynamics of tryptophan-water networks in proteins. J. Am. Chem. Soc. 2018, 140, 675-682.

(53) Draganski, A. R.; Friedman, J. M.; Ludescher, R. D. Solventslaved dynamic processes observed by tryptophan phosphorescence of human serum albumin. Biophys. J. 2017, 112, 881-891.

(54) Linden, A. H.; Franks, W. T.; Akbey, Ü.; Lange, S.; van Rossum, B. J.; Oschkinat, H. Cryogenic temperature effects and resolution upon slow cooling of protein preparations in solid state NMR. J. Biomol. NMR 2011, 51, 283-292. 